transfers (for shared learning) and in-utero transfers in the previous 24 hrs.

Results Data from 4 months showed a reduction of mean dispatch time for emergency transfers (including time critical) from 39 to $35 \mathrm{~min}$ (prior to the introduction of automation). As part of the shared learning component, 14 cases were identified and discussed with referring hospitals over the 4 months compared to $3,4,3$ cases in each of the previous year's respectively. The mean time of return of equipment after repair reduced from 31.3 to 9.8 days.

Conclusion By locating some available resources at a single point of time we observed positive changes in a number of measurable parameters. With this validation now we are building a dynamic real time platform (LocANTS) as proof of concept to show all the available resources ( including clinical parameters ) required for neonatal transfer in one consolidated screen alongside videoconferencing replacing the current audioconference, so that the team are ready to despatch 'time to roll' immediately the call for a transfer is made which will significantly improve KPI, handover quality, shared learning opportunities across the region, leading to efficient improved patient care.

\section{G124(P) JUNIOR DOCTORS AS QUALITY AND SAFETY LEADS}

A Yassaee, T Hodder, M Alam. Neonatal Department, Newham University Hospital, Barts Health, London, UK

\subsection{6/archdischild-2020-rcpch.98}

Aims Improve departmental approach to quality and safety (Q $+S$ ) issues, reporting culture and frontline staff's engagement with quality improvement through appointment of junior doctor $\mathrm{Q}+\mathrm{S}$ leads.

Methods Two junior doctors used the Model for Improvement. Cycle 1: Creating an issues log on the bottom of the handover list, enabling any member of the clinical team to $\mathrm{Q}$ $+\mathrm{S}$ issues. New items were discussed during handover. Issues included significant events, Datixes, and positive episodes which warranted promotion. Cycle 2: Introducing a weekly, minuted departmental meeting, chaired by a trainee. The meeting ran through the issue log, discussed complex cases, and would highlight opportunities for quality improvement. Cycle 3: Creating an online Greatix portal to encourage staff to highlight good practice, coupled with a monthly newsletter. Cycle 4: Formalising the role across changeover in order to ensure sustainability. This included a formal job description, advertisements to incoming trainees and negotiating protected time for individual(s) to carry out the role.

Results The weekly meetings supported multidisciplinary working and promoted the discussion of quality and safety issues in everyday clinical activities. The role has brought significant benefit the department's functioning and it was felt a good use of resource to offer protected time to an incoming trainee. There was good engagement with the Greatix system with 30 submitted and 3 newsletters produced. As a result of the structured approach in the weekly meetings, other trainees felt empowered to lead on their own quality improvement projects, including work with maternity and pathology services. Due to their knowledge, the $\mathrm{Q}+\mathrm{S}$ leads would often be asked to represent the department at governance/management meetings and their suggestions have led to projects in other departments.
Conclusion Junior doctors can be effective $Q+S$ advocates and leaders. Senior buy-in is essential for trainees to flourish in this role. Experience or training in leadership and/or QI is beneficial.

\section{G125(P) PASSING THE BATON: IMPROVING COMPETENCE AND CONFIDENCE IN HANDING OVER}

${ }^{1} \mathrm{D}$ Roberts, ${ }^{2} \mathrm{C}$ Lethaby. 'Department of Paediatric Medicine, Leeds Children's Hospital, Leeds, UK; ${ }^{2}$ Department of Paediatric Haematology and Oncology, Leeds Children's Hospital, Leeds, UK

\subsection{6/archdischild-2020-rcpch.99}

Introduction Clinical handover is crucial for safe, effective care of patients, and an opportunity for staff development and team building. A survey of medical staff in a tertiary children's hospital revealed that whilst 60\% have received structured training or feedback on effective handover at some point in their career, only $41 \%$ felt they received adequate training to hand over effectively. Confidence levels were rated on average at 53\%, and respondents recognised a need for further training.

Aims Improve competence and confidence of paediatric staff in handing over.

Methods Two structured, facilitator-led, interactive, group training sessions were developed and offered to paediatric staff utilising 'Plan, Do, Study, Act' (PDSA) cycles. Participants covered material on published handover guidelines, completed focused tasks designed to develop key skills which underpin handover (e.g. distilling pertinent information from case notes, prioritising workload), and drew these together to deliver structured written and verbal handovers using a validated tool (Situation, Background, Assessment, Recommendation [SBAR]). Guided discussion and reflection were promoted, and personalised verbal feedback given to participants.

Results A total of 28 attendances were recorded across 26 members of staff including junior doctors, advanced nurse practitioners, and physician associates. Anonymous feedback was sought from participants on the quality of training. Following the sessions $94.4 \%$ and $100 \%$ of attendees, respectively, who responded either agreed or strongly agreed that they felt more confident in the subject of handing over. Participants identified good aspects of training as it being practical, interactive, realistic, not critical, and encouraging. Challenges included having sufficient protected time for staff to attend and tailoring the sessions to the experience and seniority of attendees.

Conclusion This project has shown that structured, interactive training sessions have scope to increase the confidence of paediatric medical staff in handing over. Training requires that facilitators and participants have adequate, protected time to attend, and may, therefore, warrant inclusion in regional training days. Staff will be surveyed within six months to assess the longer-term impact of these training sessions on competence.

\section{G126(P) ABSTRACT WITHDRAWN}

